\title{
Computed tomographic angiography criteria in the diagnosis of brain death-comparison of sensitivity and interobserver reliability of different evaluation scales
}

\author{
Marcin Sawicki • R. Bohatyrewicz • K. Safranow • A. Walecka • J. Walecki • O. Rowinski • \\ J. Solek-Pastuszka • Z. Czajkowski • M. Guzinski • M. Burzynska • J. Wojczal
}

Received: 30 December 2013 / Accepted: 8 April 2014 / Published online: 7 May 2014

(C) The Author(s) 2014. This article is published with open access at Springerlink.com

\begin{abstract}
Introduction The standardized diagnostic criteria for computed tomographic angiography (CTA) in diagnosis of brain death (BD) are not yet established. The aim of the study was to compare the sensitivity and interobserver agreement of the three previously used scales of CTA for the diagnosis of BD. Methods Eighty-two clinically brain-dead patients underwent CTA with a delay of $40 \mathrm{~s}$ after contrast injection. Catheter angiography was used as the reference standard. CTA results were assessed by two radiologists, and the diagnosis of BD was established according to 10-, 7-, and 4-point scales.

Results Catheter angiography confirmed the diagnosis of $\mathrm{BD}$ in all cases. Opacification of certain cerebral vessels as indicator of BD was highly sensitive: cortical segments of the middle cerebral artery $(96.3 \%)$, the internal cerebral vein $(98.8 \%)$, and the great cerebral vein $(98.8 \%)$. Other vessels
\end{abstract}

M. Sawicki $(\bowtie) \cdot$ A. Walecka

Department of Diagnostic Imaging and Interventional Radiology, Pomeranian Medical University, Clinical Hospital No1, Unii

Lubelskiej 1, Szczecin 71252, Poland

e-mail: msaw@pum.edu.pl

R. Bohatyrewicz $\cdot$ J. Solek-Pastuszka

Clinic of Anesthesiology and Intensive Care, Pomeranian Medical

University, Szczecin, Poland

\section{K. Safranow}

Department of Biochemistry and Medical Chemistry, Pomeranian Medical University, Szczecin, Poland

J. Walecki

The Centre of Postgraduate Medical Education, Warsaw, Poland

\section{O. Rowinski}

2nd Department of Clinical Radiology, Medical University of

Warsaw, Warsaw, Poland were less sensitive: the pericallosal artery $(74.4 \%)$, cortical segments of the posterior cerebral artery $(79.3 \%)$, and the basilar artery $(82.9 \%)$. The sensitivities of the 10-, 7-, and 4point scales were $67.1,74.4$, and $96.3 \%$, respectively $(p<0.001)$. Percentage interobserver agreement in diagnosis of BD reached $93 \%$ for the 10 -point scale, $89 \%$ for the 7 point scale, and $95 \%$ for the 4-point scale $(p=0.37)$.

Conclusions In the application of CTA to the diagnosis of $\mathrm{BD}$, reducing the assessment of vascular opacification scale from a 10- to a 4-point scale significantly increases the sensitivity and maintains high interobserver reliability.

Keywords Brain death · Ancillary test $\cdot$ Computed tomographic angiography $\cdot$ Catheter angiography . Interobserver reliability

\author{
Z. Czajkowski \\ Regional Joint Hospital, Szczecin, Poland
}

M. Guzinski

Department of General Radiology, Interventional Radiology and Neuroradiology, Wroclaw Medical University, Wroclaw, Poland

M. Burzynska

Department of Anesthesiology and Intensive Therapy, Wroclaw Medical University, Wroclaw, Poland

J. Wojczal

Department of Neurology, Medical University of Lublin, Lublin, Poland 


\section{Introduction}

The use of instrumental tests to confirm brain death (BD) is still the subject of debate [1-3]. In North America, confirmatory testing is not mandatory while in certain European, Central and South American, and Asian countries it is an obligatory part of the procedure to establish BD. Nevertheless, ancillary tests are useful when specific components of the clinical examination cannot be reliably performed. Currently, the most commonly used tests in Europe include electroencephalography (EEG), evoked potentials, transcranial Doppler (TCD), perfusion scintigraphy using ${ }^{99 \mathrm{~m}} \mathrm{Tc}-\mathrm{HMPAO}$ or ${ }^{99 \mathrm{~m}} \mathrm{Tc}-\mathrm{ECD}$, and catheter cerebral angiography. However, all of them have drawbacks related to their feasibility and availability. Electrophysiological tests are susceptible to confounding factors. EEG is unreliable in barbiturate overdose or deep anesthesia and vulnerable to the hostile electrical environment of the ICU. Evoked potentials generally can only be applied in cases of primary supratentorial or secondary brain injuries, because in isolated infratentorial processes they can produce false positive results. Blood flow studies such as TCD, perfusion scintigraphy, and catheter angiography are more feasible but less available in many countries. Therefore, new imaging methods such as magnetic resonance techniques (angiography, spectroscopy, and diffusion-weighted imaging) and positron emission tomography have been proposed [4-7].

Recently, computed tomographic angiography (CTA) attracted attention as a new method for the diagnosis of BD. It appears to be a promising diagnostic alternative that is feasible, widely available, non-invasive, and expeditious. In 1998, Dupas et al. reported the first application of CTA to the diagnosis of BD [8]. Since then, several major studies of this application were published, and national guidelines were introduced in several countries (e.g., in France, Austria, Switzerland, the Netherlands, and Canada) [9-12]. Nevertheless, CTA is not widely accepted as an ancillary test for the diagnosis of BD. The main obstacle is insufficient diagnostic confidence of this method [13]. Until recently, there were no standardized criteria for interpretation of CTA results. The previous major studies assessing CTA in the diagnosis of BD showed that stasis of contrast in the proximal segments of cerebral arteries is frequently found in brain-dead patients, and this phenomenon does not preclude the diagnosis of BD. Based on those observations, three evaluation systems have been proposed: 10-, 7-, and 4-point scales. They include different numbers of intracranial vessels of anterior and posterior cerebral circulation like the 10-point scale or solely anterior circulation like the 7and 4-point scales. The common criterion of BD in each scale is a lack of opacification of cortical branches of the middle cerebral arteries (MCA-M4) and the deep cerebral veins.

Another issue related to the application of CTA refers to potential difficulty in the assessment of intracranial filling in patients with suspected BD. As previous studies using CTA showed intracranial vessels in these patients are markedly thinner, and their opacification, regardless it's level, is significantly weaker than normal [14]. Moreover, cerebral vessels are frequently obscured by subarachnoid hemorrhage (SAH) or increased density of subarachnoid space due to pseudosubarachnoid hemorrhage (pseudoSAH) phenomenon, which are frequently found in this group of patients. This can cause difficulties in determining the level of intracranial opacification and produce discrepant diagnoses of BD among radiologists. Therefore, it is reasonable to question interobserver reliability of CTA, especially with the 4-point scale, in which the diagnosis of BD is based on the analysis of only four vessels. Reliability was not addressed in the previous studies, even though it can be useful in the implementation of CTA for the diagnosis of BD.

The aim of the present study was to compare the sensitivity and reliability of the three evaluation scales in CTA for the diagnosis of BD. For this purpose, a single-phase CTA with a delay of $40 \mathrm{~s}$ was used. Sensitivity of CTA was compared to the gold standard method-catheter angiography.

\section{Material and methods}

\section{Study population}

In the prospective, multicenter study, 82 patients were examined.

The inclusion criteria were as follows:

1. Deep unresponsive coma (GCS 3-5) of an established etiology capable of causing neurological death.

2. Absence of brainstem reflexes:

a. Pupils mid-position or greater and absent pupillary light reflex (fixed dilated pupils),

b. Spontaneous eye movements,

c. Facial muscle movement to a noxious stimulus,

d. Corneal,

e. Gag/pharyngeal,

f. Cough/tracheal,

g. Oculo-cephalic

h. Vestibulo-ocular ('cold caloric').

The exclusion criteria were unresuscitated shock (mean arterial blood pressure $\leq 80 \mathrm{mmHg}$ ) and hypothermia (defined as surface temperature $\leq 35^{\circ} \mathrm{C}$ ). These are known depressants of cerebral blood flow.

The study population consisted of 45 men and 37 women with a mean age of 52.2 years (range, 22-78 years). Initial causes of coma were traumatic brain injury $(n=16)$, intracerebral hemorrhage $(n=32)$, subarachnoid hemorrhage $(n=21)$, ischemic stroke $(n=7)$, and anoxia $(n=6)$. Decompressive 
craniectomy was performed in 26 (32\%) patients prior to examinations. All patients were managed in the ICUs of two Polish university hospitals and one multi-profile provincial hospital. All subjects underwent cerebral CTA followed by catheter angiography. During all examinations, patients were normoventilated, and mean arterial blood pressure was maintained above $80 \mathrm{mmHg}$. The elapsed time between the finding of complete brainstem areflexia and the beginning of the radiological examination ranged from 6 through $48 \mathrm{~h}$ (not always recorded). Longer delays were usually caused by a prolonged initial observation period due to prior use of sedatives. Shortly, after the radiological studies, the complete clinical testing for determination of $\mathrm{BD}$ was performed. According to the Polish national legal regulations, $\mathrm{BD}$ was declared when coma, brainstem areflexia, and absence of a breathing drive with 10 -min $\mathrm{CO}_{2}$ challenge (apnea test) were confirmed. The apnea test result was positive (i.e., supported the clinical diagnosis of $\mathrm{BD}$ ) if respiratory movements were absent and arterial $\mathrm{PCO}_{2}$ was $60 \mathrm{mmHg}$ (or $20 \mathrm{mmHg}$ increase in arterial $\mathrm{PCO}_{2}$ over a baseline normal arterial $\mathrm{PCO}_{2}$ ). The clinical test had to be performed twice. Brain death was declared by a commission of three specialists, including at least one in the field of anesthesiology and intensive care and one in the field of neurology or neurosurgery. The majority of brain-dead patients became organ donors. In some cases, organ donation was not possible for medical reasons and occasionally because of a failure to obtain permission from relatives. In such situations, families were informed about the termination of futile therapy, and ventilators were legally discontinued.

\section{CTA}

Two scanners were used to perform CTA: Sensation 64 (Siemens, Erlangen, Germany) and Discovery CT 750 HD (GE Healthcare, Millwaukee, USA). CTA was preceded by a nonenhanced CT scan, which served as a reference. After injecting the contrast, scans were performed with a fixed delay of $40 \mathrm{~s}$. A bolus tracking technique was not used to ensure that this CTA protocol was widely applicable nationwide; this option is not available in all Polish CT facilities. The detailed protocol is presented in Table 1.

In the proposed protocol, $80 \mathrm{ml}$ of Iomeron 400 (Bracco Imaging, Konstanz, Germany) was injected at a flow rate of $4 \mathrm{ml} / \mathrm{s}$. This gave injection duration of $20 \mathrm{~s}$. To determine the appropriate delay of scanning, a time to peak concentration of contrast in the head and neck arteries was calculated from the formula proposed by Bae [15]:

$\mathrm{T}_{\text {peak }}=\mathrm{T}_{\text {injection }}+8 \mathrm{~s}=20 \mathrm{~s}+8 \mathrm{~s}=28 \mathrm{~s}$

$\mathrm{T}_{\text {peak }} \quad$ Time to peak concentration of contrast in the head and neck arteries

$\mathrm{T}_{\text {injection }}$ Injection duration.
Table 1 Technical parameters of the CTA methods used for diagnosis of $\mathrm{BD}$

\begin{tabular}{|c|c|c|c|c|}
\hline \multirow[t]{2}{*}{ Parameter } & \multicolumn{2}{|c|}{$\begin{array}{l}\text { Siemens Somatom } \\
\text { Sensation } 64\end{array}$} & \multicolumn{2}{|c|}{$\begin{array}{l}\text { GE Discovery CT } \\
750 \mathrm{HD}\end{array}$} \\
\hline & NECT & CTA & NECT & CTA \\
\hline \multicolumn{5}{|l|}{ Acquisition } \\
\hline Scan range & \multicolumn{4}{|c|}{$\mathrm{C} 5 / \mathrm{C} 6$ to vertex } \\
\hline Tube voltage (KVp) & 120 & 100 & 120 & 120 \\
\hline Tube current (mAs) & 380 & 160 & 320 & 160 \\
\hline Rotation time (s) & 1.0 & 0.5 & 1.0 & 0.6 \\
\hline Collimation (mm) & $20 \times 0.6$ & $64 \times 0.6$ & $32 \times 0.625$ & $32 \times 0.625$ \\
\hline Feed (mm/rotation) & 4.8 & 23.0 & 16 & 26.25 \\
\hline Pitch factor & 0.8 & 1.2 & 0.8 & 1.2 \\
\hline Scan time (s) & 35 & 6 & 10 & 6 \\
\hline \multicolumn{5}{|l|}{ Reconstruction } \\
\hline Slice width (mm) & 5.0 & 0.6 & 2.5 & 0.6 \\
\hline Increment (mm) & 5.0 & 0.4 & 2.5 & 0.6 \\
\hline Field of view (mm) & 250 & 250 & 250 & 250 \\
\hline Matrix & $512 \times 512$ & $512 \times 512$ & $512 \times 512$ & $512 \times 512$ \\
\hline RECON algorithm & $\mathrm{H} 31 \mathrm{~s}$ & H10f & Standard & Standard \\
\hline \multicolumn{5}{|l|}{ Contrast injection } \\
\hline Start delay (s) & & 40 & & 40 \\
\hline Volume (ml) & & 80 & & 80 \\
\hline Flow rate $(\mathrm{ml} / \mathrm{s})$ & & 4 & & 4 \\
\hline Injection duration (s) & & 20 & & 20 \\
\hline
\end{tabular}

After adding $15 \mathrm{~s}$ to accommodate possible slowed cerebral circulation due to increased cerebrovascular resistance and subtracting a time needed for scanning from the level of $\mathrm{C} 5$ / C6 to the circle of Willis, the final formula is as follows:

$\mathrm{T}_{\text {delay }}=\mathrm{T}_{\text {peak }}+15 \mathrm{~s}-\frac{\mathrm{T}_{\text {scan }}}{2}=28 \mathrm{~s}+15 \mathrm{~s}-3 \mathrm{~s}=40 \mathrm{~s}$

$\mathrm{T}_{\text {delay }} \quad$ Delay of CTA scanning after contrast injection $\mathrm{T}_{\text {scan }}$ Scan duration.

All CTA examinations were evaluated by two radiologists, blinded to each other's assessments, to the results of the clinical test and to the results of the catheter angiography. They had 4 and 8 years of experience interpreting cerebral angiography.

\section{CTA criteria of BD}

Opacification of superficial temporal arteries was assessed to confirm that the contrast was injected correctly and to detect possible hemodynamic perturbations. The following 10 intracranial vessels were evaluated:

- Pericallosal segments of the right and left anterior cerebral artery (ACA-A3), 
- Cortical segments of the right and left middle cerebral artery (MCA-M4),

- Cortical segments of the right and left posterior cerebral artery (PCA-P2),

- Basilar artery (BA),

- Right and left internal cerebral vein (ICV),

- Great cerebral vein (GCV) - the vein of Galen.

A point was given for each vessel without opacification, resulting in scores between 0 and 10 . Diagnosis of BD was established according to the three previously used evaluation systems: 10-, 7-, and 4-point scales-see Fig. 1. Positive result in each scale confirming the diagnosis of BD was noted with scores 10,7 , and 4 , respectively.

Catheter cerebral angiography

All patients underwent catheter angiography as the second part of the radiological evaluation. Angiography was performed from $15 \mathrm{~min}$ to $3 \mathrm{~h}$ after CTA. Three angiographic systems were used: Axiom Artis, Fluorospot Top (Siemens, Erlangen, Germany), and MultiDiagnost 3 (Philips, Eindhoven, the Netherlands). The same technique was applied as described previously [16-18]. A typical femoral approach was used in all cases. In 67 patients, aortocervical angiography was used. After positioning a Pigtail 4-5 F catheter in the ascending aorta, $30 \mathrm{ml}$ of contrast was injected at a flow rate of $15 \mathrm{ml} / \mathrm{s}$. In the remaining 15 patients, carotid and vertebral arteries were catheterized selectively; $8 \mathrm{ml}$ of contrast was injected into each artery at a flow rate of $8 \mathrm{ml} / \mathrm{s}$. We visualized the head and neck by recording 50-s series with a frequency of $2 \mathrm{f} / \mathrm{s}$ and using a digital subtraction technique. A local neuroradiologist performed and assessed all studies.

\section{Angiographic criteria of BD}

Cerebral circulatory arrest was diagnosed by catheter angiography if either of the following criteria were met:
- Non-filling of intracranial vessels with the normal flow in the external carotid arteries,

- Stasis filling - delayed, weak, and persistent opacification of the proximal cerebral arterial segments without opacification of the cortical branches or venous outflow.

Data collection

Demographic data recorded for brain-dead patients were age and sex. Clinical data were initial cause of coma, the presence of craniectomy, SAH, or pseudoSAH. PseudoSAH is wellknown phenomenon of increased attenuation of the subarachnoid space mimicking SAH. For the diagnosis of BD in CTA and catheter angiography, opacification of the following vessels was recorded:

- Superficial temporal arteries (STA),

- Pericallosal segments of the right and left anterior cerebral artery (ACA-A3),

- Cortical segments of the right and left middle cerebral artery (MCA-M4),

- Cortical segments of the right and left posterior cerebral artery (PCA-P2),

- Basilar artery (BA),

- Right and left internal cerebral vein (ICV),

- Great cerebral vein (GCV) - the vein of Galen.

The presence of stasis filling in CTA and catheter angiography was also noted. Stasis filling was defined as opacification of one or more of the following arterial segments:

- $\mathrm{ACA}-\mathrm{A} 1$ and $\mathrm{A} 2$,

- MCA-M1, M2, and M3,

- PCA-P1 without opacification of the cortical arterial branches or deep veins.

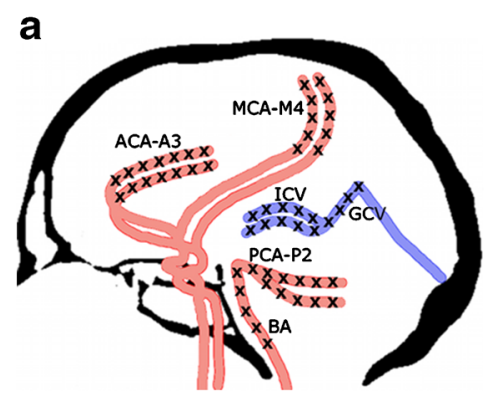

b

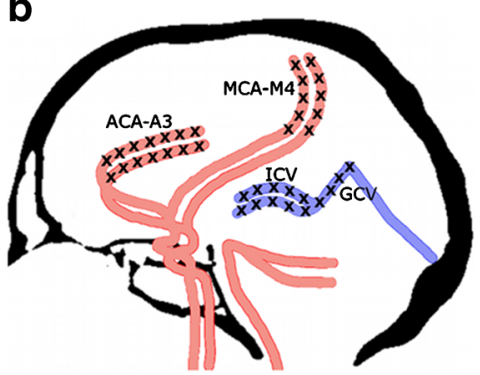

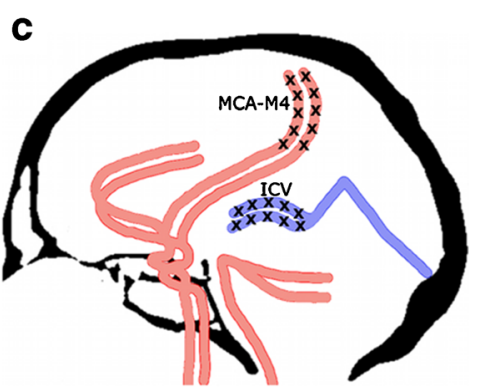

Fig. 1 Different criteria for the diagnosis of BD by CTA: a Positive result in the 10-point scale (score=10) confirming the diagnosis of $\mathrm{BD}$ was recorded when the following vessels were not opacified: the bilateral PCA-P2, the BA, the bilateral ACA-A3, the bilateral MCA-M4, the bilateral ICV, and the GCV. Scores from 0 to 9 were classified as negative results excluding the diagnosis of $\mathrm{BD}$; $\mathbf{b}$ In the 7-point scale, positive result (score $=7$ ) was recorded with a lack of opacification of the bilateral ACA-A3, the bilateral MCA-M4, the bilateral ICV, and the GCV. Scores from 0 to 6 were classified as negative results; $\mathbf{c}$ Positive result in the 4point scale $($ score $=4)$ was recorded when the bilateral MCA-M4 and the bilateral ICV were not opacified. Scores from 0 to 3 were classified as negative results 
Two radiologists independently evaluated each CTA and recorded the diagnosis of $\mathrm{BD}$ using the 10-, 7-, and 4-point scales. A false negative result of CTA was noted when the test precluded BD otherwise confirmed by catheter angiography and clinical testing. A true positive result of CTA was recorded when CTA, catheter angiography, and clinical testing concordantly confirmed BD.

\section{Statistical analysis}

Interobserver agreement was measured by the Kendall's tau-b rank correlation coefficient for dichotomous variables (diagnosis of BD). The significance of interobserver differences was assessed by McNemar's test. Cochran's Q test was used to compare the proportion of false negative results of $\mathrm{BD}$ diagnosis between scales. A multivariate logistic regression model with sex, age, presence of craniectomy, and occurrence of $\mathrm{SAH} / \mathrm{pseudoSAH}$ as independent variables was used to find independent predictors of false negative CTA results. $P<0.05$ was considered statistically significant. STATISTICA 10 software (StatSoft, Tulsa, USA) was used for the statistical analyses. The tests were evaluated by a medical statistician.

\section{Results}

In all 82 cases, clinical tests confirmed BD. Catheter angiography results were consistent with the diagnosis of $\mathrm{BD}$ in all cases, revealing no intracranial filling in $62(75.6 \%)$ patients with the normal flow in the external carotid arteries. Stasis filling was observed in $20(24.4 \%)$ patients. This phenomenon was observed from 4 to $48 \mathrm{~s}$ after intraarterial injection. No venous outflow was observed in any case.

The analysis of interobserver agreement did not reveal significant differences between the two radiologists who assessed CTA for the diagnosis of BD; see Table 2.

There were 19 discordant diagnoses of $\mathrm{BD}$ between radiologists analyzing CTA. Both radiologists jointly revised each discrepant finding to reach a consensus, which is presented in the following CTA results.

All CTA examinations revealed opacification of the STA with no discrepancies noted between radiologists (percentage agreement was $100 \%$ ).

Table 2 Interobserver agreement in the diagnosis of $\mathrm{BD}$

\begin{tabular}{llll}
\hline Scale & tau-b & Percentage agreement & $p$ value \\
\hline 10-point & 0.84 & 93 & $0.68(\mathrm{~ns})$ \\
7-point & 0.71 & 89 & $0.50(\mathrm{~ns})$ \\
4-point & 0.64 & 95 & $0.13(\mathrm{~ns})$ \\
\hline
\end{tabular}

Note: $p$ for systematic difference between radiologists (McNemar's test); $n s$ not significant
Analysis of opacification of particular cerebral vessels in CTA revealed that they could be classified into the following two groups:

- Frequently opacified vessels: the ACA-A3 (in 21 patients), PCA-P2 (17), and BA (14),

- Rarely opacified vessels: MCA-M4 (3 patients), ICV (1), and GCV (1), see Table 3.

Regarding their sensitivity as indicators of cerebral circulatory arrest, the high sensitivity group included cortical segments of the MCA, ICV, and GCV. The low sensitivity group consisted of the pericallosal artery and cortical segments of the PCA and BA. The sensitivities of all six vessels differed significantly $(p<0.001$, Cochran's Q test). There were no significant differences between vessels within the high sensitivity group and the low sensitivity group, see Fig. 2.

According to the 10-point scale, 27 false negative CTA results were observed, see Fig. 3. With the 7-point scale, the number of false negative results decreased to 21, see Fig. 4. The 4-point scale provided three false negative results, see Fig. 5. A comparison of sensitivities for different CTA scales is presented in Fig. 6.

According to the 10-point scale, CTA showed a non-filling pattern in 32 (39\%) patients and stasis filling in $23(28 \%)$ patients.

Sex, age, presence of craniectomy, and the occurrence of $\mathrm{SAH} /$ pseudoSAH were analyzed as independent predictors of a false negative CTA result in the 10-point scale. Presence of craniectomy appeared to be an independent predictive factor of a false negative CTA. Relative risk of a false negative CTA in patients with craniectomy was 3.13 times higher than in those without it ( $95 \% \mathrm{CI}=1.70$ to $5.77 ; p=0.0002)$. The second independent predictor of a false negative CTA was the presence of SAH/pseudoSAH observed in 54 patients. Relative risk of a false negative CTA in patients with SAH/ pseudoSAH was 2.98 times higher than in those without it ( $95 \% \mathrm{CI}=1.14$ to $7.78 ; p=0.0255$ ). Sex and age were not associated with false negative CTA results.

\section{Discussion}

The percents of agreement between the radiologists were equally high (89-95\%) for all scales. The fact that the observers did not reach perfect agreement could be explained by significantly lower contrast enhancement of cerebral arteries in brain-dead patients compared to normal opacification [14]. However, the analysis of interobserver agreement showed a trend towards lower values of Kendall's tau-b coefficient using the 4-point scale. Interobserver agreement coefficients, including tau-b, are affected by the prevalence of measures under consideration; therefore, low values of tau-b may be due to rare findings, such as negative CTA results using the 4- 


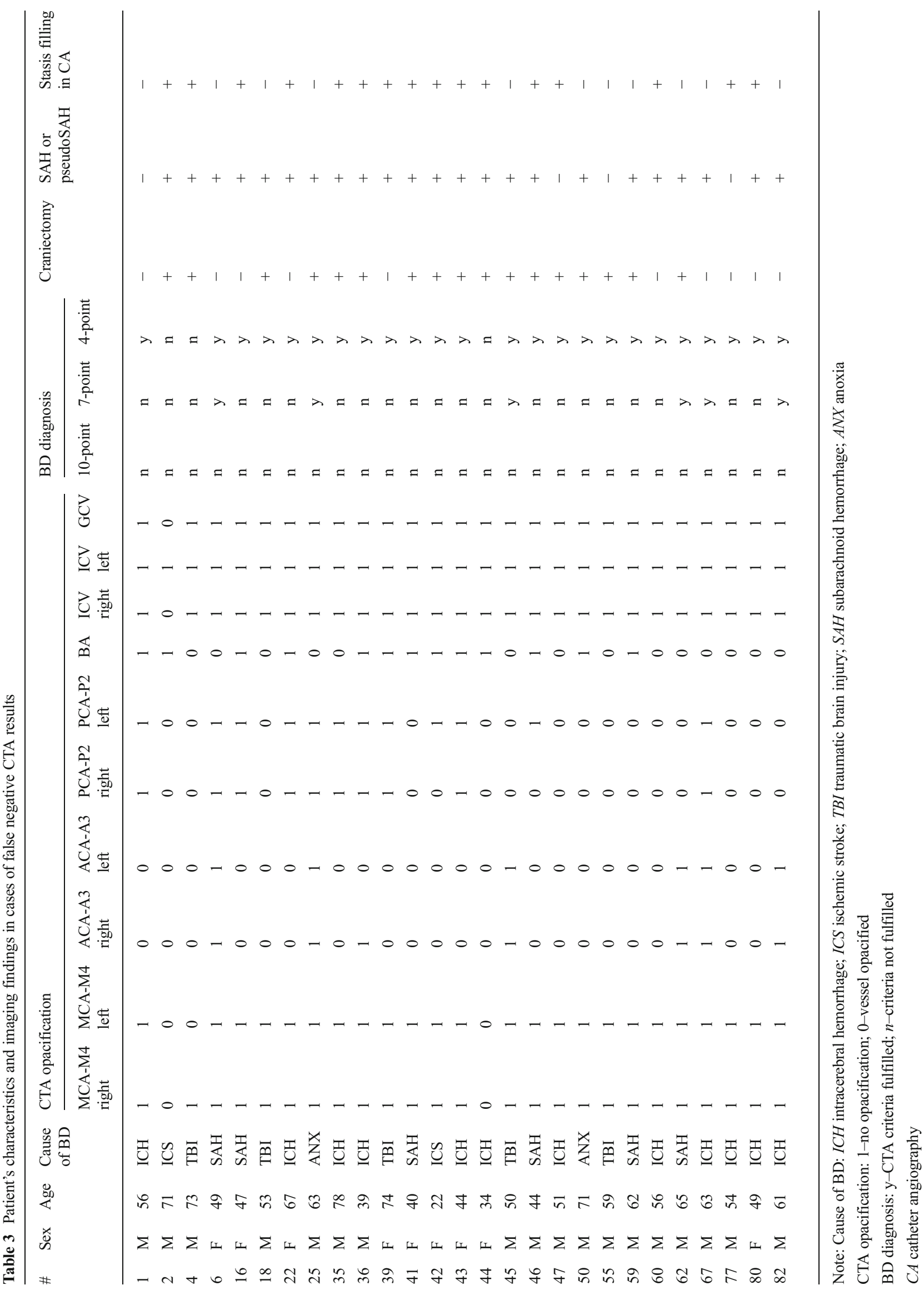




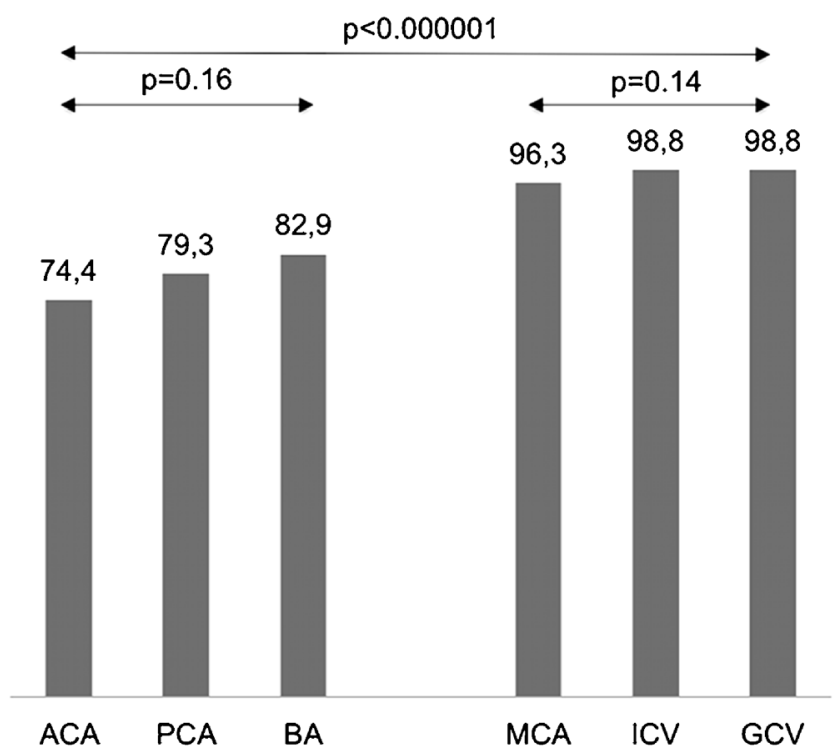

Fig. 2 Sensitivity of cerebral vessels as indicators of cerebral circulatory arrest in CTA. All vessels were classified into the high sensitivity group (cortical segments of the MCA, ICV, and GCV) and the low sensitivity group (the pericallosal artery and cortical segments of the PCA and BA)

point scale, may not reflect low rates of agreement. This is a known paradox explained by Feinstein and Cicchetti $[19,20]$. In such situations, the percentage agreement is more reliable indicator of interobserver concordance.

Concerning opacification of individual cerebral vessels as indicators of cerebral circulatory arrest, two groups can be distinguished:

- High sensitivity: MCA, ICV, and GCV,

- Low sensitivity: ACA, PCA, and BA.

In previous studies, different sets of vessels were considered in the diagnosis of cerebral circulatory arrest [8, 21-26].
The first evaluation scale was introduced by Dupas et al. in 1998 [8]. They observed $100 \%$ sensitivity and specificity of the pericallosal artery, MCA-M4, ICV, and GCV, and proposed the 7-point system, which excluded arteries of the posterior cerebral circulation, because their sensitivity was lower ( $85.7 \%$ for BA). Delayed opacification of the BA in clinically brain-dead patients was observed in several studies using catheter angiography [27-31]. Braun et al. reported this phenomenon in $24(17 \%)$ of 140 patients meeting clinical criteria of BD; these findings were verified by positive TCD in six cases [31]. Only four previous studies assessed opacification of the BA with CTA in BD [8, 21, 23, 25]. Combes et al. reported a $100 \%$ sensitivity of the BA [25]; however, the other authors observed sensitivities that ranged from 86 to $94 \%$. These later results are consistent with our finding for the BA, $82.9 \%$ sensitivity. Simultaneously, in all cases, catheter angiography showed a lack of opacification of BA in the 30- to 50-s series. In the study by Welschehold et al., all cases were verified by a positive TCD result [21]. These findings show that opacification of BA can be observed in up to $17 \%$ of braindead patients and does not indicate preserved cerebral perfusion, thus does not preclude diagnosis of BD. A high frequency of BA opacification in brain-dead patients can be explained by the protective role of the cerebellar tentorium. The majority of primary brain injuries affect the supratentorial compartment; therefore, increasing intracranial pressure (ICP) primarily stops capillary and venous flow to the cerebral hemispheres. In these injuries, ICP may rise more slowly in the infratentorial region, allowing contrast to enter arteries of posterior cerebral circulation. Cessation of flow in posterior cranial fossa can occur with a delay, providing false negative results of blood flow examinations. One of such cases is presented in Fig. 3. a

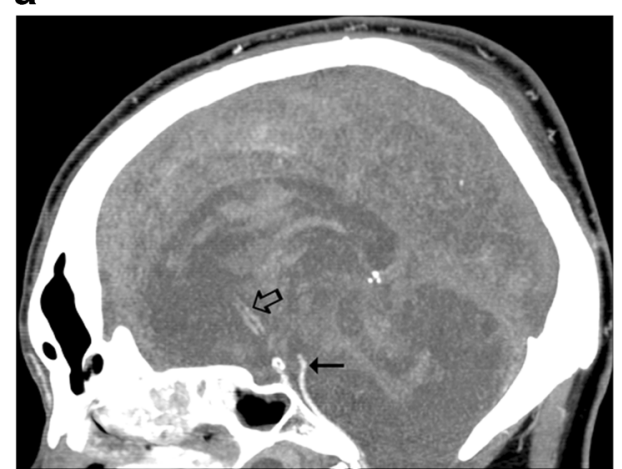

b

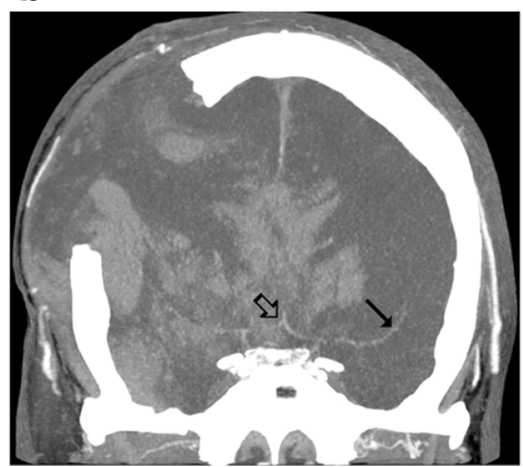

c

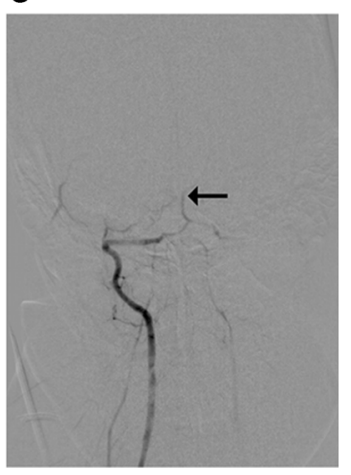

Fig. 3 CTA findings in a 50-year-old man (patient no. 45) with traumatic brain injury (epidural hematoma in the right parietal region, massive intracerebral, and subarachnoid and intraventricular hemorrhage) and right sided craniectomy presented with signs of BD on clinical examination: a Ten millimeter maximum intensity projection (MIP) in sagittal plane. CTA shows opacification of the BA (thin arrow) and a trace of contrast in A2 segments of the ACAs (thick arrow). b Ten millimeter MIP in coronal plane. CTA shows opacification of the M1 segment of the left MCA (thin arrow) and the A1 segments of the ACAs (thick arrow); these findings exclude the diagnosis of $\mathrm{BD}$ according to the 10-point scale but confirm BD according to the 7- and 4-point scales. $\mathrm{c}$ Catheter angiography of the right VA performed $0.5 \mathrm{~h}$ later revealed delayed, residual filling of the BA (arrow) that occurred $21 \mathrm{~s}$ after injection. This result was interpreted as stasis filling consistent with the diagnosis of BD 
a

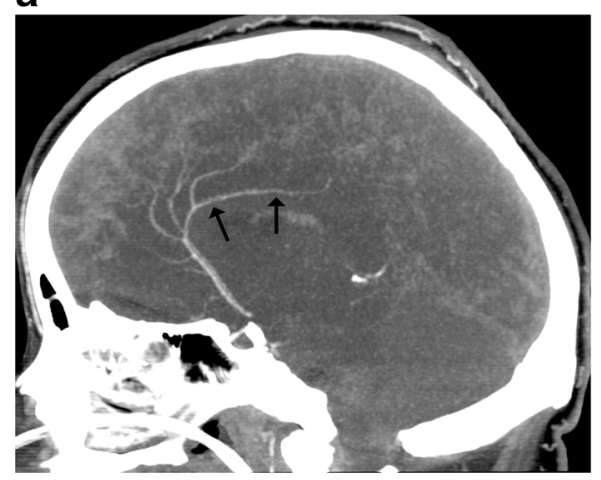

Fig. 4 CTA findings in a 22-year-old woman (patient no. 42) with a brain stem ischemic stroke and a right sided craniectomy who presented with signs of BD on clinical examination. a Ten millimeter MIP in sagittal plane. CTA shows opacification of the right pericallosal artery (thin arrows); b Ten millimeter MIP in coronal plane. CTA shows opacification of the M1 segments of the MCAs (thin arrows). These findings exclude

In regard to the diagnostic value of pericallosal artery, the $100 \%$ sensitivity observed by Dupas et al. was not confirmed in other studies that reported a sensitivity of $57-83 \%$ for this artery $[8,21-25]$. In this study, the pericallosal artery had the lowest level among all the vessels evaluated. The shorter distance from the skull base to the pericallosal artery, in comparison with the MCA-M4, can explain the lower sensitivity of the ACA-A3 for the CTA diagnosis of BD. According to Poisseuille's equation, cerebral blood flow (CBF) is inversely proportional to the length of the vessel.

$\mathrm{CBF}=\frac{\pi \times \mathrm{CPP} \times \text { radius }^{4}}{8 \times \text { viscosity } \times \text { length }}$

CPP Cerebral perfusion pressure

Radius Radius of the vessel
C

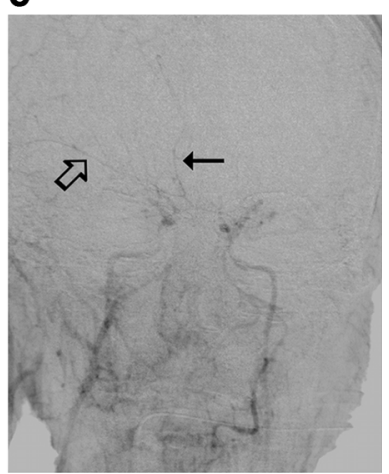

the diagnosis of $\mathrm{BD}$ according to the 10- and 7-point scales but confirm $\mathrm{BD}$ according to the 4-point scale. $\mathrm{c}$ Catheter angiography from the aortic arch performed $1 \mathrm{~h}$ later revealed delayed, residual filling of the M1 segment of the right MCA (thick arrow) and A2 segment of the right ACA (thin arrow) that occurred $32 \mathrm{~s}$ after injection. This result was interpreted as stasis filling consistent with the diagnosis of BD

$\begin{array}{ll}\text { Viscosity } & \text { Viscosity of the blood } \\ \text { Length } & \text { Length of the vessel. }\end{array}$

Therefore, raised ICP primarily stops the flow in the distal cortical segments of the MCA, whereas the contrasts column continues to reach more proximal pericallosal arteries for some time. One such case is presented in Fig. 4.

A high frequency of opacification of ACA-A3 in braindead patients is the principal cause of the low sensitivity of CTA in the 10- and 7-point scales previously reported and confirmed by this study, see Table 4 .

In 2006, Leclerc et al. introduced the 4-point scale, which is limited to the most sensitive parameters, the MCA and ICV, and excludes the ACA and GCV [26]. Exclusion of GCV was motivated by the fact that it drains blood partially from the posterior circulation. Therefore, this was a consequence of exclusion of the BA and PCA. In addition, circulation to the

a

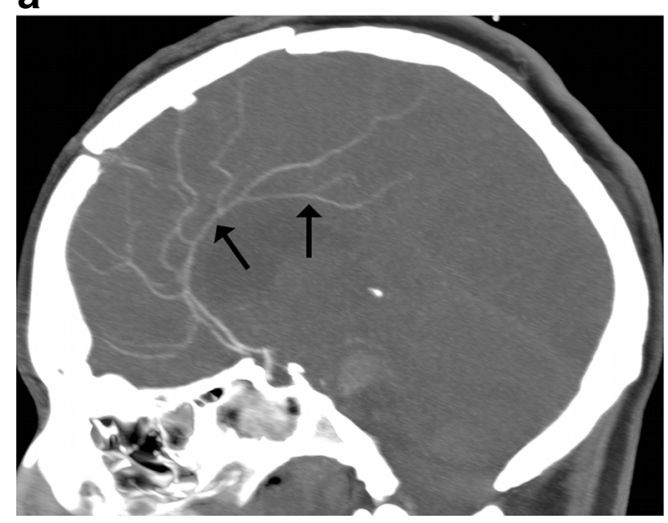

Fig. 5 CTA findings in a 34-year-old woman (patient no. 44) with brain stem hematoma and frontal craniotomy presenting signs of BD on clinical examination. a Ten millimeter MIP in sagittal plane. CTA shows opacification of both pericallosal arteries (thin arrows); b Ten millimeter MIP in coronal plane. CTA shows opacification of the cortical segments of the MCAs (thin arrows); these findings exclude the diagnosis of BD b

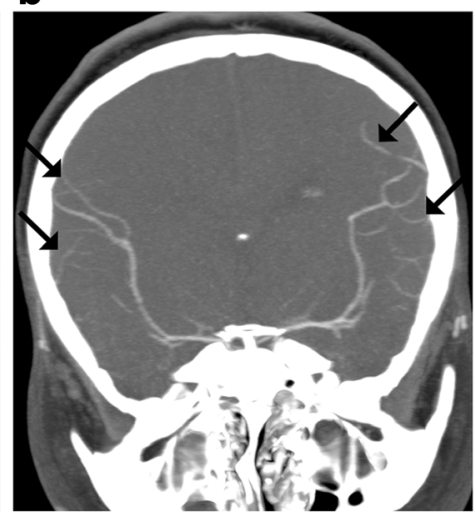

according to the 10-, 7-, and 4-point scales. c Catheter angiography of the right ICA performed $3 \mathrm{~h}$ later revealed delayed, residual filling of the M1 segments of the MCAs (thin arrows) and the A2 segments of the ACAs (thick arrow) that occurred $18 \mathrm{~s}$ after injection. This result was interpreted as stasis filling consistent with the diagnosis of $\mathrm{BD}$ 


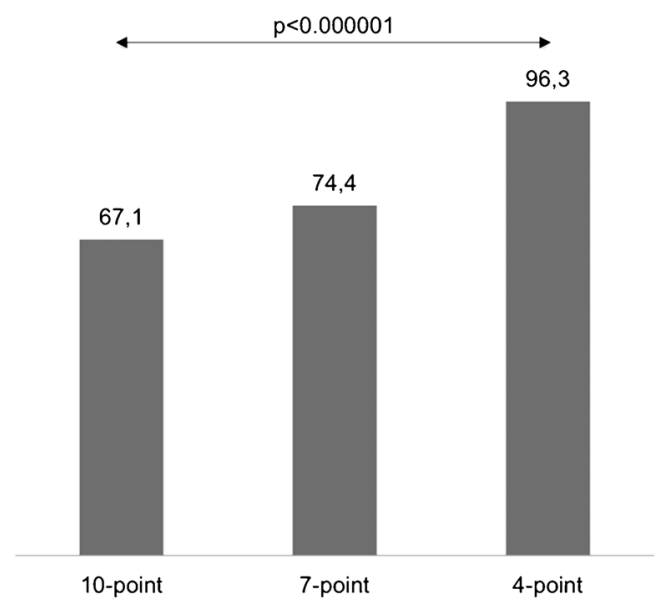

Fig. 6 Sensitivity of three CTA scales for the diagnosis of BD. The sensitivities differed significantly ( $p<0.001$, Cochran's Q test)

basal ganglia is assessed by opacification of the ICVs; therefore, opacification of the GCV, a confluence of the ICVs, does not provide any additional information, despite its high individual sensitivity. This approach was included in the recommendations of the French Society of Neuroradiology. The GCV was included in their earlier, 2007, recommendations, but was excluded from their most recent, 2011, revised recommendations [12, 32]. To summarize, evaluation of CTA in the diagnosis of $\mathrm{BD}$ evolved from the 10- and 7-point systems to the more highly sensitive 4-point scale.

It must be noted that respecting the widely accepted concept of the whole brain death, an ancillary test should assess the perfusion of the entire brain including anterior and posterior circulation. This condition is not fulfilled in the 7- and 4point scales limited to anterior circulation. However, some established ancillary tests do not entirely address this issue either. TCD for the diagnosis of $\mathrm{BD}$ consists of assessing blood flow in MCAs and BA, omitting ACAs and PCAs.

Table 4 Results of the major studies that evaluated the sensitivity of CTA for the diagnosis of BD by the type of scale utilized

\begin{tabular}{llll}
\hline Study authors and year & \multicolumn{2}{l}{ Sensitivity (\%) } & \\
\hline & 10-point & 7-point & 4-point \\
Combes et al. 2007 [25] & 70 & & \\
Welschehold et al. * 2013 [21] & 54 & & \\
Dupas et al. 1998 [8] & & 100 & \\
Quesnel et al. ** 2007 [24] & & 52 & \\
Frampas et al. 2009 [23] & & 63 & 86 \\
Rieke et al. 2011 [22] & & 76 & 93 \\
Leclerc et al. 2006 [26] & 67.1 & 74.4 & 96.3 \\
Present study & & & 87 \\
\hline
\end{tabular}

Note: *GCV was not assessed, **This study included 5 (of 21) patients with anoxic brain injury, which predisposes to preserved residual perfusion
There are opinions that in planar radionuclide perfusion examination it is difficult to evaluate the perfusion of the brainstem because of overlying parotid gland and other soft tissues [33]. Evoked potentials allow examination of specific areas of interest in the brainstem. Finally, EEG records activity from only the cortical layers immediately beneath the scalp; it does not record from subcortical structures, such as the brainstem or thalamus. It should be considered that ancillary test, even if limited to assessment of the anterior circulation, is always combined with the clinical examination testing brainstem function. Besides, no false positive result of CTA according to 7- or 4-point scale has been reported so far (i.e., CTA confirmed BD, yet the patient survived).

While analyzing a relationship between demographic and clinical features, and the sensitivity of CTA, it was found that craniectomy independently predisposed to false negative results. Preserved intracranial opacification in brain-dead patients with skull defects, particularly craniectomy, was previously reported [22, 23, 30, 31, 34]. Three of such cases are presented in Figs. 3, 4, and 5. This can be explained by regional reduction of ICP caused by a skull defect. Alvarez et al. postulated that intracranial hypertension is nevertheless severe enough to induce cerebral circulatory arrest in these cases [30]. However, the residual cerebral flow at the region of the skull defect is a major limitation of perfusion tests for confirmation of BD. Frampas et al. recommended a minimum observation period of $6 \mathrm{~h}$ before CTA in this clinical situation because of the slow increase of ICP [23].

$\mathrm{SAH}$ or pseudoSAH appeared to be a second factor that predisposed to false negative CTA results. This finding may be explained by increased baseline density of the subarachnoid space in patients with $\mathrm{SAH}$ or pseudoSAH, which can be mistaken for a true vascular opacification. These findings support the observation of Gutierrez et al. that interpretation of CT can be difficult in such circumstances [35].

In many patients, catheter angiography showed more proximal intracranial opacification than did CTA. Dupas et al. observed the same divergence in four of seven cases [8]. A larger volume of contrast was used in CTA than in catheter angiography, but during angiography, contrast was injected intra-arterially, which minimizes the dilutional effect. This discrepancy could have resulted from the sequence in which CTA and catheter angiography were performed. Angiography was performed from $15 \mathrm{~min}$ to $3 \mathrm{~h}$ after CTA. During this time interval, a slowly rising ICP could further suppress cerebral circulation and stop contrast movement at more proximal levels.

The proposed CTA protocol differs from the standard technique of CTA in the diagnosis of BD consisting of NECT and two post-contrast phases started at 20 and $60 \mathrm{~s}$ after injection [36]. The 20- to 60 -s protocol was proposed by Dupas et al. in 1998 [8] and was applied in several later studies [21-26]. However, in clinical practice, particularly in France, 
it is routinely combined with whole body CT for assessing transplantable organs with a single contrast injection. Such combination requires a larger volume of contrast $(120 \mathrm{ml}$ instead of $\approx 80 \mathrm{ml}$ ) and longer injection duration ( $40 \mathrm{~s}$ instead of $\approx 20 \mathrm{~s}$ ) in comparison to a standard CTA of the brain.

The technique proposed in the present study is dedicated for assessing cerebral vessels, as whole body CT was not included in the examination. Therefore, a volume of contrast was reduced to $80 \mathrm{ml}$ - a standard for cerebral CTA. A standard flow rate of $4 \mathrm{ml} / \mathrm{s}$ was used as well. This gave injection duration of $20 \mathrm{~s}$ - twice as shorter in comparison to the 20- to 60 -s protocol. While choosing a proper delay of CTA scanning for the diagnosis of $\mathrm{BD}$, one must take into account that in some cases cerebral circulation may be slowed due to intracranial hypertension. A key issue is to cover this delayed vascular opacification with CTA scanning. As perfusion and angiographic studies show, there is a threshold time of approximately $15 \mathrm{~s}$ for cerebral circulation to preserve a viability of the brain [28, 37-41]. With longer circulation time, an opacification of cerebral vessels does not indicate preserved brain perfusion thus does not preclude the diagnosis of $\mathrm{BD}$. Thus, optimal CTA scan for the diagnosis of BD should be performed approximately $15 \mathrm{~s}$ after peak concentration of contrast is achieved in the head and neck arteries. This time is $40 \mathrm{~s}$ after injection with the injection duration of $20 \mathrm{~s}$ used in the present study. It should be noted that the standard 60 -s phase is also performed approximately $15 \mathrm{~s}$ after peak concentration of contrast in the head and neck arteries, because the standard protocol includes the 40-s injection duration.

Applying the injection duration of $20 \mathrm{~s}$ and following the standard protocol with scanning at $60 \mathrm{~s}$ after injection would be consistent with assessing vascular opacification over $30 \mathrm{~s}$ after peak contrast concentration in the head and neck arteries. This would detect extremely slow cerebral circulation not indicative of preserved perfusion but providing false negative results and decreasing the sensitivity of CTA.

Another difference between the proposed protocol and the 20 - to 60-s technique is an abandonment of the early 20 -s phase. The early phase is used for assessing of opacification of extracranial arteries as an indicator of sufficient contrast delivery. However, the study by Leclerc et al. showed that extracranial arteries are similarly opacified in the 20-s and in the 60 -s phase [26]. They postulated that the scanning protocol could be limited to a single, 60 -s phase. Therefore, it was reasonable to assume that scanning with a delay of $40 \mathrm{~s}$ will be sufficient to detect opacification of extracranial arteries. The results confirmed these assumptions because all CTA examinations revealed sufficient opacification of the STA. The proposed single-phase protocol can be a feasible alternative to the two-phase technique in the diagnosis of $\mathrm{BD}$, when whole body CT is not simultaneously performed. In comparison to the two-phase protocol, the single-phase method is simpler to perform and requires a lower volume of contrast, which is less harmful for the kidneys. However, the accuracy of both protocols needs to be comparatively evaluated.

\section{Limitations}

The study was limited to one measure, sensitivity, of the accuracy of CTA for the diagnosis of BD. To more completely describe the accuracy of CTA for the diagnosis of BD, assessment of its specificity is needed, preferably with a control group, including comatose patients at different degrees of intracranial hypertension.

\section{Conclusions}

In the application of $\mathrm{CTA}$ for the diagnosis of $\mathrm{BD}$, reducing the assessment of vascular opacification scale from a 10- to a 4-point scale significantly increases the sensitivity and maintains high interobserver reliability. Therefore, the 4-point scale seems to be optimal in the determination of BD. Nevertheless, introduction of CTA as reliable ancillary test cannot be recommended without evaluation of its specificity, which has not been comprehensively studied yet.

Ethical Standards and Patient Consent We declare that all human and animal studies have been approved by the local ethics committee and have therefore been performed in accordance with the ethical standards laid down in the 1964 Declaration of Helsinki and its later amendments. We declare that all patients/guardians gave informed consent prior to inclusion in this study.

Acknowledgments This study was supported by a grant from the State Committee for Scientific Research of Poland No N N403 171137.

Conflict of interest We declare that we have no conflict of interest.

Open Access This article is distributed under the terms of the Creative Commons Attribution License which permits any use, distribution, and reproduction in any medium, provided the original author(s) and the source are credited.

\section{References}

1. Young GB, Shemie SD, Doig CJ, Teitelbaum J (2006) Brief review: the role of ancillary tests in the neurological determination of death. Can J Anaesth=J Can Anesth 53(6):620-627. doi:10.1007/ BF03021855

2. Wijdicks EF (2010) The case against confirmatory tests for determining brain death in adults. Neurology 75(1):77-83. doi:10.1212/ WNL.0b013e3181e62194

3. Roberts DJ, MacCulloch KA, Versnick EJ, Hall RI (2010) Should ancillary brain blood flow analyses play a larger role in the neurological determination of death? Can J Anaesth=J Can Anesth 57(10): 927-935. doi:10.1007/s12630-010-9359-4

4. Aichner F, Felber S, Birbamer G, Luz G, Judmaier W, Schmutzhard E (1992) Magnetic resonance: a noninvasive approach to metabolism, 
circulation, and morphology in human brain death. Ann Neurol 32(4):507-511. doi:10.1002/ana.410320405

5. Ishii K, Onuma T, Kinoshita T, Shiina G, Kameyama M, Shimosegawa Y (1996) Brain death: MR and MR angiography. AJNR Am J Neuroradiol 17(4):731-735

6. Selcuk H, Albayram S, Tureci E, Hasiloglu ZI, Kizilkilic O, Cagil E, Kocer N, Islak C (2012) Diffusion-weighted imaging findings in brain death. Neuroradiology 54(6):547-554. doi:10.1007/s00234-011-0912-9

7. Meyer MA (1996) Evaluating brain death with positron emission tomography: Case report on dynamic imaging of $18 \mathrm{~F}$ fluorodeoxyglucose activity after intravenous bolus injection. $\mathrm{J}$ Neuroimaging: Off J Am Soc Neuroimaging 6(2):117-119

8. Dupas B, Gayet-Delacroix M, Villers D, Antonioli D, Veccherini MF, Soulillou JP (1998) Diagnosis of brain death using two-phase spiral CT. AJNR Am J Neuroradiol 19(4):641-647

9. Brain Death Protocol (2006). Health Council of the Netherlands

10. Shemie SD, Lee D, Sharpe M, Tampieri D, Young B, Canadian Critical Care S (2008) Brain blood flow in the neurological determination of death: Canadian expert report. Can J Neurol Sci Le J Canadien Sci Neurol 35(2):140-145

11. The Determination of Death in the Context of Organ Transplantation. Medical-ethical guidelines (2011). Swiss Academy of Medical Sciences

12. de Neuroradiologie SF, de Radiologie SF, de la Biomedecine A (2011) Recommendations on diagnostic criteria of brain death by the technique of CT angiography. J Neuroradiol J Neuroradio 38(1): 36-39. doi:10.1016/j.neurad.2011.01.001

13. van der Lugt A (2010) Imaging tests in determination of brain death. Neuroradiology 52(11):945-947. doi:10.1007/s00234-010-0765-7

14. Sawicki M, Bohatyrewicz R, Safranow K, Walecka A, Walecki J, Rowinski O, Solek-Pastuszka J, Czajkowski Z, Marzec-Lewenstein E, Motyl K, Przybyl W, Czarnecka A (2013) Dynamic evaluation of stasis filling phenomenon with computed tomography in diagnosis of brain death. Neuroradiology 55(9):1061-1069. doi:10.1007/s00234013-1210-5

15. Bae KT (2010) Intravenous contrast medium administration and scan timing at CT: Considerations and approaches. Radiology 256(1):3261. doi:10.1148/radiol.10090908

16. Bohatyrewicz R, Sawicki M, Walecka A, Walecki J, Rowinski O, Bohatyrewicz A, Kanski A, Czajkowski Z, Krzysztalowski A, SolekPastuszka J, Zukowski M, Marzec-Lewenstein E, Wojtaszek M (2010) Computed tomographic angiography and perfusion in the diagnosis of brain death. Transplant Proc 42(10):3941-3946. doi: 10.1016/j.transproceed.2010.09.143

17. Sawicki M, Walecka A, Bohatyrewicz R, Poncyljusz W, Kordowski J (2010) Computed Tomographic Angiography in the diagnosis of brain death. Med Sci Monit 16(suppl 1):28-32

18. Sawicki M, Walecka A, Kordowski J, Poncyljusz W, Gabrysz-Trybek E, Maczka M, Bohatyrewicz R (2009) Computed tomographic angiography in the evaluation of brain death. Pol J Radiol 74(2):22-27

19. Feinstein AR, Cicchetti DV (1990) High agreement but low kappa: I. The problems of two paradoxes. J Clin Epidemiol 43(6):543-549

20. Cicchetti DV, Feinstein AR (1990) High agreement but low kappa: II. Resolving the paradoxes. J Clin Epidemiol 43(6):551-558

21. Welschehold S, Kerz T, Boor S, Reuland K, Thomke F, Reuland A, Beyer C, Wagner W, Muller-Forell W, Giese A (2013) Detection of intracranial circulatory arrest in brain death using cranial CTangiography. Eur J Neurol: Off J Eur Fed Neurol Soc 20(1):173179. doi:10.1111/j.1468-1331.2012.03826.x

22. Rieke A, Regli B, Mattle HP, Brekenfeld C, Gralla J, Schroth G, Ozdoba C (2011) Computed tomography angiography (CTA) to prove circulatory arrest for the diagnosis of brain death in the context of organ transplantation. Swiss Med Wkly 141:w13261. doi:10.4414/ smw.2011.13261

23. Frampas E, Videcoq M, de Kerviler E, Ricolfi F, Kuoch V, Mourey F, Tenaillon A, Dupas B (2009) CT angiography for brain death diagnosis. AJNR Am J Neuroradiol 30(8):1566-1570. doi:10.3174/ ajnr.A1614

24. Quesnel C, Fulgencio JP, Adrie C, Marro B, Payen L, Lembert N, El Metaoua S, Bonnet F (2007) Limitations of computed tomographic angiography in the diagnosis of brain death. Intensive Care Med 33(12):2129-2135. doi:10.1007/s00134-007-0789-6

25. Combes JC, Chomel A, Ricolfi F, d'Athis P, Freysz M (2007) Reliability of computed tomographic angiography in the diagnosis of brain death. Transpl Proc 39(1):16-20. doi:10.1016/j. transproceed.2006.10.204

26. Leclerc X, Taschner CA, Vidal A, Strecker G, Savage J, Gauvrit JY, Pruvo JP (2006) The role of spiral CT for the assessment of the intracranial circulation in suspected brain-death. J Neuroradiol J Neuroradiol 33(2):90-95

27. Bradac GB, Simon RS (1974) Angiography in brain death. Neuroradiology 7(1):25-28

28. Rosenklint A, Jorgensen PB (1974) Evaluation of angiographic methods in the diagnosis of brain death. Correlation with local and systemic arterial pressure and intracranial pressure. Neuroradiology 7(4):215-219

29. Kricheff II, Pinto RS, George AE, Braunstein P, Korein J (1978) Angiographic findings in brain death. Ann N Y Acad Sci 315:168183

30. Alvarez LA, Lipton RB, Hirschfeld A, Salamon O, Lantos G (1988) Brain death determination by angiography in the setting of a skull defect. Arch Neurol 45(2):225-227

31. Braun M, Ducrocq X, Huot JC, Audibert G, Anxionnat R, Picard L (1997) Intravenous angiography in brain death: Report of 140 patients. Neuroradiology 39(6):400-405

32. Leclerc X (2007) CT angiography for the diagnosis of brain death: Recommendations of the French Society of Neuroradiology (SFNR). J Neuroradiol J Neuroradiol 34(4):217-219. doi:10.1016/j.neurad. 2007.07.008

33. Zuckier L, Kolano J (2008) Radionuclide studies in the determination of brain death: Criteria, concepts, and controversies. Semin Nucl Med 38(4):262-273. doi:10.1053/j.semnuclmed.2008.03.003

34. Escudero D, Otero J, Marques L, Parra D, Gonzalo JA, Albaiceta GM, Cofino L, Blanco A, Vega P, Murias E, Meilan A, Roger RL, Taboada F (2009) Diagnosing brain death by CT perfusion and multislice CT angiography. Neurocrit Care 11(2):261-271. doi:10. 1007/s12028-009-9243-7

35. Gutierrez LG, Rovira A, Portela LA, Leite Cda C, Lucato LT (2010) $\mathrm{CT}$ and MR in non-neonatal hypoxic-ischemic encephalopathy: Radiological findings with pathophysiological correlations. Neuroradiology 52(11):949-976. doi:10.1007/s00234-010-0728-z

36. Taylor T, Dineen R, Gardiner D, Buss C, Howatson A, Chuzhanova N, Pace N (2012) Computed tomography (CT) angiography for confirmation of the clinical diagnosis of brain death (Protocol). Cochrane Database Syst Rev Issue 3. doi:10.1002/14651858.CD009694

37. Carrera E, Jones PS, Iglesias S, Guadagno JV, Warburton EA, Fryer TD, Aigbirhio FI, Baron JC (2011) The vascular mean transit time: a surrogate for the penumbra flow threshold? J Cereb Blood Flow Metab: Off J Int Soc Cereb Blood Flow Metab 31(4):1027-1035. doi:10.1038/jcbfm.2010.197

38. Abels B, Klotz E, Tomandl BF, Kloska SP, Lell MM (2010) Perfusion CT in acute ischemic stroke: a qualitative and quantitative comparison of deconvolution and maximum slope approach. AJNR Am J Neuroradiol 31(9):1690-1698. doi:10.3174/ajnr.A2151

39. Dankbaar JW, de Rooij NK, Rijsdijk M, Velthuis BK, Frijns CJ, Rinkel GJ, van der Schaaf IC (2010) Diagnostic threshold values of cerebral perfusion measured with computed tomography for delayed cerebral ischemia after aneurysmal subarachnoid hemorrhage. Stroke; J Cereb Circ 41(9):1927-1932. doi:10.1161/STROKEAHA. 109.574392

40. Sparacia G, Iaia A, Assadi B, Lagalla R (2007) Perfusion CT in acute stroke: Predictive value of perfusion parameters in assessing tissue 
viability versus infarction. Radiol Med 112(1):113-122. doi:10.1007/ s11547-007-0125-9

41. Sobesky J, Zaro Weber O, Lehnhardt FG, Hesselmann V, Thiel A, Dohmen C, Jacobs A, Neveling M, Heiss WD (2004) Which time-to- peak threshold best identifies penumbral flow? A comparison of perfusion-weighted magnetic resonance imaging and positron emission tomography in acute ischemic stroke. Stroke; J Cereb Circ 35(12):2843-2847. doi:10.1161/01.STR.0000147043.29399.f6 